\title{
On the Structure of the Bose-Einstein Condensate Ground State
}

\author{
A. I. Solomon*, Y. Feng ${ }^{\dagger}$, and V. Penna ${ }^{\ddagger}$ \\ Faculty of Mathematics and Computing, \\ The Open University, Milton Keynes, MK7 6AA, United Kingdom
}

(August 18, 2019)

We construct a macroscopic wave function that describes the Bose-Einstein condensate and weakly excited states, using the $s u(1,1)$ structure of the mean-field hamiltonian, and compare this state with the experimental values of second and third order correlation functions.

PACS numbers: 03.75.Fi, 05.30.Jp, 32.80.Pj

The recent experimental achievement [1] of Bose-Einstein condensation (BEC) has stimulated a great revival of interest in the theoretical study of this phenomenon. One fascinating aspect of the Bose-Einstein Condensate is the nature of coherence in a macroscopic quantum system, and in recent experiments some of the coherence properties of BEC have been discussed and explicitly addressed [2, [n this paper, we describe the hamiltonian and energy eigenstates within the su(1,1) mean-field picture of BEC and, based on this theory, we construct a generalised version of the BEC weakly excited states. We calculate some correlation functions within this theory, and compare with recent experimental results.

The standard description of the Bose-Einstein condensate is by means of an order parameter field $\Psi(\mathbf{x})$ which accounts locally for the physical state of the system [5]. The hamiltonian has the standard form

$$
\begin{aligned}
\mathcal{H}[\Psi]= & \int d^{3} x\left[\frac{\hbar^{2}}{2 m}|\nabla \Psi(\mathbf{x})|^{2}+U(\mathbf{x})|\Psi(\mathbf{x})|^{2}\right] \\
& +\frac{1}{2} \int d^{3} x \int d^{3} y \Psi^{*}(\mathbf{y}) \Psi^{*}(\mathbf{x}) V(\mathbf{x}, \mathbf{y}) \Psi(\mathbf{y}) \Psi(\mathbf{x}) .
\end{aligned}
$$

Representing the field $\Psi(\mathbf{x})$ by its Fourier transformation leads to the second quantized form

$$
\mathcal{H}=\sum_{k} \epsilon_{k} n_{k}+\frac{1}{2} \sum_{k} \sum_{p, q} V_{k} a_{p+k}^{+} a_{q-k}^{+} a_{p} a_{q}
$$

where $V_{k} \equiv V(|\mathbf{k}|)$, is a momentum preserving interaction. The number operators $n_{k} \equiv a_{k}^{+} a_{k}$, the raising operators $a_{k}^{+}$, and the lowering operators $a_{l}$ obey the Weyl-Heisenberg algebra commutators

$$
\left[a_{l}, a_{k}^{+}\right]=\delta_{l k} \mathbf{I},\left[a_{l}^{+}, n_{k}\right]=-\delta_{l k} a_{k}^{+},\left[a_{l}, n_{k}\right]=\delta_{l k} a_{k}
$$

The Bogoliubov prescription is that at zero temperature the state with $k=0$ is macroscopically occupied and this observation allows one to treat $a_{0}^{+}$and $a_{0}$ as c-numbers $\left(\left[a_{0}, a_{0}^{+}\right] \simeq 0\right)$ since the corresponding number operator $n_{0}$, counting the bosons constituting the condensate, turns out to be macroscopically large. However this neglect of the operators $a_{0}^{+}$and $a_{0}$ is not an appropriate approximation if we wish to describe phenomena in the condensate ground

\footnotetext{
*Email: a.i.solomon@open.ac.uk

${ }^{\dagger}$ Email: y.feng@open.ac.uk

${ }^{\ddagger}$ Permanent address: Dipartimento di Fisica, Politecnico di Torino, Corso Duca degli Abruzzi 24, I-10129 Torino, Italy. Email: penna@athena.polito.it
} 
states. So here we no longer adopt such an approximation and we retain the operator status of $a_{0}^{+}$and $a_{0}$ in order to give a more consistent description of the state of the condensed system.

Making explicit the terms depending on $a_{0}$ and $a_{0}^{+}$

in (2) and neglecting those terms that contain three or four boson operators $a_{k}^{+}, a_{l}(k, l \neq 0)$ reduces $\mathcal{H}$ to the form

$$
\begin{aligned}
\mathcal{H}= & \epsilon_{0} n_{0}+\frac{V_{0}}{2} a_{0}^{+2} a_{0}^{2}+\sum_{k \neq 0} \epsilon_{k} n_{k}+n_{0} V_{0} \sum_{k \neq 0} n_{k} \\
& +\frac{1}{2} \sum_{k \neq 0} V_{k}\left[n_{0}\left(n_{k}+n_{-k}\right)+a_{0}^{2} a_{k}^{+} a_{-k}^{+}+a_{0}^{+2} a_{k} a_{-k}\right]
\end{aligned}
$$

where $\epsilon_{k}=\hbar^{2} k^{2} / 2 m$ ( $m$ is the fluid atom mass).

The hamiltonian is linearized by using the mean-field approximation procedure which reduces bilinear operators such as $A B$ to the linear form

$$
A B \simeq A\langle B\rangle+\langle A\rangle B-\langle A\rangle\langle B\rangle
$$

based on the assumption $(A-\langle A\rangle)(B-\langle B\rangle) \simeq 0$. We note that a similar approach, starting instead from a bosonic Hubbard model and using a novel form of this linearisation procedure [6], leads to essentially to the same $s u(1,1)$ structure for the excited states, as well as an additional condensate term for the ground state. This yields the quadratic reduced hamiltonian

$$
\mathcal{H}_{m f}=\sigma_{0}\left(n_{0}+\frac{1}{2}\right)+\frac{1}{2}\left(u_{0} a_{0}^{+2}+u_{0}^{*} a_{0}^{2}\right)+\sum_{k \neq 0} \mathcal{H}^{(k)}-E_{*}
$$

where

$$
\begin{gathered}
\sigma_{0} \equiv \epsilon_{0}+\frac{1}{2} \sum_{k \neq 0}\left(V_{0}+V_{k}\right)\left(\left\langle n_{k}\right\rangle+\left\langle n_{-k}\right\rangle\right), \quad u_{0} \equiv V_{0}\left\langle a_{0}^{2}\right\rangle+\sum_{k \neq 0} V_{k}\left\langle a_{k} a_{-k}\right\rangle \\
E_{*}=\frac{1}{2}\left[V_{0}\left|\left\langle a_{0}^{+2}\right\rangle\right|^{2}+\sigma_{0}\right]+\frac{1}{2} \sum_{k \neq 0}\left[\left(\sigma_{k}-\epsilon_{k}\right)\left\langle n_{k}+n_{-k}\right\rangle+\sigma_{k}\right] \\
+\frac{1}{2} \sum_{k \neq 0}\left(u_{k}\left\langle a_{k}^{+} a_{-k}^{+}\right\rangle+u_{k}^{*}\left\langle a_{k} a_{-k}\right\rangle\right),
\end{gathered}
$$

and the pair mode hamiltonian $\mathcal{H}^{(k)}$ is

$$
\mathcal{H}^{(k)}=\frac{\sigma_{k}}{2}\left(n_{k}+n_{-k}+1\right)+\frac{1}{2}\left(u_{k} a_{k}^{+} a_{-k}^{+}+u_{k}^{*} a_{k} a_{-k}\right)
$$

with

$$
\sigma_{k} \equiv \epsilon_{k}+\left\langle n_{0}\right\rangle\left(V_{0}+V_{k}\right), u_{k} \equiv V_{k}\left\langle a_{0}^{2}\right\rangle .
$$

We can rewrite $\mathcal{H}_{m f}$ in the $s u(1,1)$ form

$$
\begin{aligned}
\mathcal{H}_{m f}= & 2\left[\sigma_{0} A_{3}^{(0)}+\frac{1}{2}\left(u_{0} A_{+}^{(0)}+u_{0}^{*} A_{-}^{(0)}\right)\right] \\
& +\sum_{k \neq 0}\left[\sigma_{k} A_{3}^{(k)}+\frac{1}{2}\left(u_{k} A_{+}^{(k)}+u_{k}^{*} A_{-}^{(k)}\right)\right]-E_{*}
\end{aligned}
$$


by means of the generators of the algebra $s u(1,1)$

$$
A_{3}^{(0)}=\frac{1}{2}\left(n_{0}+\frac{1}{2}\right), A_{+}^{(0)}=\frac{a_{0}^{+2}}{2}, A_{-}^{(0)}=\frac{a_{0}^{2}}{2}
$$

and

$$
A_{3}^{(k)}=\frac{1}{2}\left(n_{k}+n_{-k}+1\right), A_{+}^{(k)}=a_{k}^{+} a_{-k}^{+}, A_{-}^{(k)}=a_{k} a_{-k}
$$

that account for the momentum creation/destruction processes occurring in the fluid and involving the modes $k$ and $-k$. These satisfy the usual commutation relations

$$
\left[A_{+}^{(q)}, A_{-}^{(q)}\right]=-2 A_{3}^{(q)},\left[A_{3}^{(q)}, A_{ \pm}^{(q)}\right]= \pm A_{ \pm}^{(q)}, q=0, k,-k
$$

It is known that within the $s u(1,1)$ mean-field picture the energy eigenstates are expressed a direct product of $s u_{k}(1,1)$ coherent states [7]. We therefore write the eigenstates as

$$
|\xi\rangle=\left|\xi_{0}\right\rangle \otimes_{k \neq 0}\left|\xi_{k}\right\rangle
$$

where

$$
\left|\xi_{k}\right\rangle=\exp \left[\xi_{k} A_{+}^{(k)}-\xi_{k}^{*} A_{-}^{(k)}\right]|0\rangle
$$

with th $\xi_{k}=-u_{k} / \sigma_{k}$. The eigenvalues of $\mathcal{H}_{k}$ are given by $E_{k}=\sqrt{\sigma_{k}^{2}-\left|u_{k}\right|^{2}}$.

The factor $\left|\xi_{0}\right\rangle$ is normally absent (i.e., it is implicitly traced away) in the standard approach due to the semiclassical status of $a_{0}^{+}, a_{0}$. Here it restores the condensate to its role as a dynamically active degree of freedom, that is

$$
\left|\xi_{0}\right\rangle=\exp \left[\xi_{0} A_{+}^{(0)}-\xi_{0}^{*} A_{-}^{(0)}\right]|0\rangle
$$

where $\operatorname{th} \xi_{0}=-u_{0} / \sigma_{0}$.

Writing for brevity

$$
S\left(\xi_{k}\right) \equiv \exp \left[\xi_{k} A_{+}^{(k)}-\xi_{k}^{*} A_{-}^{(k)}\right]
$$

we may express the state $|\xi\rangle$ as

$$
|\xi\rangle=\otimes_{q} S\left(\xi_{q}\right)|0\rangle \quad(q=0,+k,-k)
$$

The operators $S\left(\xi_{k}\right)$ are similar to, but not identical with, the vacuum squeezing operators $\exp \left[\frac{1}{2}\left(\xi a^{+2}-\xi^{*} a^{2}\right)\right]$ familiar from Quantum Optics.

The structure of the state $|\xi\rangle$ clearly exhibits the imprint of the mean-field dynamical algebra $\mathcal{A}_{*}=\oplus_{k} s u_{k}(1,1)$ which provides an approximate description of the dynamical processes occurring inside the system. The main unattractive feature is the fact that

$$
\left\langle a_{0}\right\rangle \equiv\left\langle\xi\left|a_{0}\right| \xi\right\rangle=0
$$

(arising from the two-boson character of $\mathcal{A}_{*}$ ), whereas the low temperature regime should be characterised by a nonvanishing order parameter $\langle\Psi(\mathbf{x})\rangle$, or equivalently $\left\langle a_{0}\right\rangle \sqrt{V}$ (recall that $\left|\left\langle a_{0}\right\rangle / \sqrt{V}\right|^{2} \simeq N$ where $N$ is the total 
particle number inside the volume $V$ ) due to the strong depletion of the $k$ mode states. In the state $|\xi\rangle$ we also clearly have $\left\langle a_{k}\right\rangle \equiv\left\langle\xi\left|a_{k}\right| \xi\right\rangle=0$.

In addition, the values of the second order correlation function

$$
g^{(2)}(0)=\frac{\left\langle a_{0}^{+2} a_{0}^{2}\right\rangle}{\left\langle a_{0}^{+} a_{0}\right\rangle^{2}}
$$

and the third order correlation function

$$
g^{(3)}(0)=\frac{\left\langle a_{0}^{+3} a_{0}^{3}\right\rangle}{\left\langle a_{0}^{+} a_{0}\right\rangle^{3}}
$$

for the states $|\xi\rangle$ do not agree with the experimental results, which seem to indicate that $g^{(2)}(0)$ and $g^{(3)}(0)$ are not exactly equal to 1 , but slightly larger than one [3, 4 . However it is easy to show that $g^{(2)}(0)=1$ and $g^{(3)}(0)=1$ in the state $D(\alpha)|0\rangle(D$ state $)$ if the mean density $\left\langle n_{0}\right\rangle$ is a large number, where $D(\alpha)=\exp \left(\alpha a_{0}^{+}-\alpha^{*} a_{0}\right)$.

These considerations motivate our attempt to generalise $|\xi\rangle$ to $|\xi, z\rangle$

$$
|\xi, z\rangle=\left|\xi_{0}, z_{0}\right\rangle \otimes_{k \neq 0}\left|\xi_{k}, z_{k}\right\rangle
$$

by introducing the further definitions

$$
\begin{aligned}
& \left|\xi_{0}, z_{0}\right\rangle=D\left(z_{0}\right)\left|\xi_{o}\right\rangle \\
& \left|\xi_{k}, z_{k}\right\rangle=D\left(z_{k}\right)\left|\xi_{k}\right\rangle,
\end{aligned}
$$

where $D\left(z_{q}\right)=\exp \left(z_{q} a_{q}^{+}-z_{q}^{*} a_{q}\right), \quad q=0, k,-k$.

We now describe the BEC states by $|\xi, z\rangle$ where

$$
|\xi, z\rangle=\otimes_{q}\left|\xi_{q}, z_{q}\right\rangle=\otimes_{q} D\left(z_{q}\right) S\left(\xi_{q}\right)|0\rangle \quad(q=0, \pm 1, \pm 2 \ldots)
$$

For obvious reasons, we refer to the state $|\xi, z\rangle$ as a $D S$ state, the $D S$ operator being similar to, but not identical with, that which produces a squeezed state in Quantum Optics, namely

$$
\exp \left(z a^{+}-z^{*} a\right) \exp \left[\frac{1}{2}\left(\xi a^{+2}-\xi^{*} a^{2}\right)\right]
$$

(for a single mode).

The BEC state (14) involves a large number of parameters $\left\{\xi_{k}, z_{k}\right\}$ which, as is usual in mean-field theories, may in principle be determined by a self-consistent treatment. However, we would expect $z_{k}=0$ for $k \neq 0$ (since there is no condensation other than in the $k=0$ state); and if we are primarily interested in condensate properties we need determine only $\xi_{0}$ and $z_{0}$ (4 real parameters). These may be calculated from the condensate conditions, as we now show. We have the following expectations:

$$
\begin{aligned}
\left\langle\xi, z\left|a_{0}\right| \xi, z\right\rangle & =\left\langle\xi_{o}\left|D^{+}\left(z_{0}\right) a_{0} D\left(z_{0}\right)\right| \xi_{o}\right\rangle=z_{0} \\
\left\langle\xi, z\left|n_{0}\right| \xi, z\right\rangle & =\left\langle\xi_{0}\left|D^{+}\left(z_{0}\right) n_{0} D\left(z_{0}\right)\right| \xi_{o}\right\rangle=\left|z_{0}\right|^{2}+\mathrm{sh}^{2}\left|\xi_{0}\right| \\
\left\langle\xi, z\left|a_{k}\right| \xi, z\right\rangle & =\left\langle\xi_{k}\left|a_{k}\right| \xi_{k}\right\rangle=z_{k} \\
\left\langle\xi, z\left|a_{k} a_{-k}\right| \xi, z\right\rangle & =\left\langle\xi_{k}\left|a_{k} a_{-k}\right| \xi_{k}\right\rangle=z_{k} z_{-k} \\
\left\langle\xi, z\left|n_{k}\right| \xi, z\right\rangle & =\left\langle\xi_{k}\left|n_{k}\right| \xi_{k}\right\rangle=\left|z_{k}\right|^{2}+\mathrm{sh}^{2}\left|\xi_{k}\right|
\end{aligned}
$$


The state $|\xi, z\rangle$ incorporates both the $s u(1,1)$ structure inherited from the spectrum-generating algebra approach to the mean field hamiltonian, as well as the nonvanishing expectation values for the operators $a_{k}$ implicit in a conventional (Heisenberg-Weyl) coherent state. As we shall show, a choice of the parameters for the state $|\xi, z\rangle$ state allows one to fit the experimental values of $g^{(2)}(0)$ and $g^{(3)}(0)$.

Ketterle and Miesner [3] pointed out that data on the condensate expansion energy, combined with spectroscopic scattering length measurements, can be used to give the second order correlation function $g^{(2)}(0)$ in alkali condensates. An experiment on a BEC of sodium [8] [9] by Ketterle et al yielded $g^{(2)}(0)=1.25 \pm 0.58$, and the experiment on a rubidium condensate [10] yielded $g^{(2)}(0)=1.0 \pm 0.2$. In another important experiment [4], Burt et al. recently compared the trap loss due to three-body recombination of a rubidium condensate to that of a thermal cloud, and obtained $7.4 \pm 2.6$ for the ratio of the third order correlation function $g^{(3)}(0)$ values in the thermal and condensed states.

Although the experimental results are not inconsistent with a pure $D$ state, at least in the case of rubidium, indications for sodium are that $g^{(2)}(0)$ and $g^{(3)}(0)$ are larger than 1 . From the structure of the states $|\xi, z\rangle$, we can see that the BEC ground state is

$$
\left|\xi_{0}, z_{0}\right\rangle=D\left(z_{0}\right) S\left(\xi_{0}\right)|0\rangle
$$

where

$$
\begin{gathered}
D\left(z_{0}\right)=\exp \left(z_{0} a_{0}^{+}-z_{0}^{*} a_{0}\right) \\
S\left(\xi_{0}\right)=\exp \left(\xi_{0} A_{+}^{(0)}-\xi_{0}^{*} A_{-}^{(0)}\right), \xi_{0}=r \exp (i \phi)
\end{gathered}
$$

with $A_{+}^{(0)}=\frac{a_{0}^{+2}}{2}, A_{-}^{(0)}=\frac{a_{0}^{2}}{2}$. We now show how to choose the parameters of the BEC ground state $\left|\xi_{0}, z_{0}\right\rangle$ to fit the experimental values of $g^{(2)}(0)$ and $g^{(3)}(0)$ cited above [3, 4 .

The unitary transformation of the operators $a_{0}$ and $a_{0}^{+}$by $D\left(z_{0}\right)$ and $S\left(\xi_{0}\right)$ is given by

$$
\begin{array}{r}
S^{+}\left(\xi_{0}\right) D^{+}\left(z_{0}\right) a_{0} D\left(z_{0}\right) S\left(\xi_{0}\right)=\mu a_{0}+\nu a_{0}^{+}+z_{0} \\
S^{+}\left(\xi_{0}\right) D^{+}\left(z_{0}\right) a_{0}^{+} D\left(z_{0}\right) S\left(\xi_{0}\right)=\mu a_{0}^{+}+\nu^{*} a_{0}+z_{0}^{*}
\end{array}
$$

where we have put

$$
\mu=\operatorname{ch} r, \nu=\exp (i \phi) \operatorname{sh} r
$$

We obtain the following mean values in the $D S$ state:

$$
\begin{aligned}
\left\langle n_{0}\right\rangle & =\left|z_{0}\right|^{2}+|\nu|^{2} \\
\left\langle a_{0}^{+2} a_{0}^{2}\right\rangle= & 3|\nu|^{4}+4\left|z_{0}\right|^{2}|\nu|^{2}+\left|z_{0}\right|^{4}+|\nu|^{2}+\mu\left(z_{0}^{2} \nu^{*}+z_{0}^{* 2} \nu\right) \\
\left\langle a_{0}^{+3} a_{0}^{3}\right\rangle= & 15|\nu|^{6}+27|\nu|^{4}\left|z_{0}\right|^{2}+9|\nu|^{2}\left|z_{0}\right|^{4}+\left|z_{0}\right|^{6}+9|\nu|^{4} \\
& +9|\nu|^{2}\left|z_{0}\right|^{2}+3\left(\left|z_{0}\right|^{2}+3|\nu|^{2}\right)\left[\mu\left(z_{0}^{2} \nu^{*}+z_{0}^{* 2} \nu\right)\right]
\end{aligned}
$$


If we write

$$
\mu\left(z_{0}^{2} \nu^{*}+z_{0}^{* 2} \nu\right)=\left|z_{0}\right|^{2} \mu|\nu| \cos \left(\phi-\phi_{z}\right), z_{0}=\left|z_{0}\right| \exp \left(i \phi_{z}\right)
$$

Then the value $g^{(2)}(0)$ for the $D S$ state is

$$
g^{(2)}(0)=1+\left[\frac{2|\nu|^{2}}{\left\langle n_{0}\right\rangle}+\frac{|\nu|^{2}+\left|z_{0}\right|^{2} \mu|\nu| \cos \left(\phi-\phi_{z}\right)}{\left\langle n_{0}\right\rangle^{2}}\right]
$$

and the value $g^{(3)}(0)$ for the $D S$ state is

$$
\begin{aligned}
g^{(3)}(0)= & +\left[\frac{6|\nu|^{2}}{\left\langle n_{0}\right\rangle}+\frac{3|\nu|^{2}\left(4|\nu|^{2}+3\right)}{\left\langle n_{0}\right\rangle^{2}}\right. \\
& \left.-\frac{4|\nu|^{6}-3\left(\left|z_{0}\right|^{2}+3|\nu|^{2}\right)\left|z_{0}\right|^{2} \mu|\nu| \cos \left(\phi-\phi_{z}\right)}{\left\langle n_{0}\right\rangle^{3}}\right]
\end{aligned}
$$

These results [Eq.(20) and Eq.(21)] are plotted in Figure 1 and Figure 2. From the figures we see that the experimental results are consistent with values of $r$ between 0 and 4 ( $r=0$ is a pure condensate $D$ state).

In this note we have constructed a state for a Bose-Einstein condensate based on the $s u(1,1)$ spectrum-generating algebra structure of the mean-field hamiltonian, and the Heisenberg-Weyl coherent state structure which gives nonvanishing boson operator expectations. It is a common feature of mean-field approximations that these give rise to the loss of conserved quantities (in our case loss of number conservation) and the consequent appearance of associated order parameters, which here are $\left\langle a_{0}>\right.$ and $\left\langle a_{k} a_{-k}\right\rangle$. This is a general property of the algebraic approach [11]. The question of loss of number conservation is considered in some detail by Girardeau [12]. However the linearisation procedure herein adopted retains the momentum-conservation properties of the original hamiltonian, as in superfluidity and superconductivity. The resulting $D S$ state is similar to a squeezed coherent state, familiar in Quantum Optics, and will undoubtedly give rise to interesting squeezing phenomena which will be explored later. After completion of this work, it has been brought to our attention that a similar $D S$ description of the condensate was also obtained by Navez [13] from slightly different premises. In this note we showed that the $D S$ state provides better fits to the experimental results on the correlation functions associated with the BEC state. Although it might be argued that the additional freedom inherent in the extra parameters associated with the $D S$ state must give better fits to the experiments, it should be noted that the changes to the coherent state values (of unity) are in one direction only (positive) and are therefore only consistent with experimental values greater than one.

[1] M. H. Anderson, J.R. Ensher, M.R. Matthews, C.E. Wieman, and E.A. Cornell, Science 269, 198(1995); K. B. Davis, M.O. Mewes, M.R. Andrew, N.J. Vandruten, D.S. Durfee, D.M. Kurn, and W. Ketterle, Phys. Rev. Lett. 75, 3969(1995); C. C. Bradley, C.A. Sackett, and R.G. Hulet, Phys. Rev. Lett. 78, 985(1997)

[2] M.R. Andrews, C.G. Townsend, H.J. Miesner, D.S. Durfee, D.M. Kurn, and W Ketterle, Science 275, 637(1997)

[3] W. Ketterle and H. J. Miesner, Phys. Rev. A, 56, 3291(1997)

[4] E.A. Burt, R. W. Ghrist, C. J. Myatt, M. J. Holland, E. A. Cornell, and C. E. Wieman, Phys. Rev. Lett. 79, 337(1997) 
[5] Walter Greiner, "Quantum Mechanics. Special Chapters", Springier -Verlag Berlin Heidelberg(1998).

[6] L. Amico, M. Rasetti, and R. Zecchina, Physica A, 230, 300(1996)

[7] A. I. Solomon, Journal of Mathematical Physics 12, 390(1971).

[8] M.O. Mewes, M. R. Andrews, N. J. Vandruten, D. M. Kurn, D. S. Durfee, and W. Ketterle, Phys. Rev. Lett. 77, 416(1996)

[9] E. Tiersinga, C. J. Williams, P. S. Julienne, K. M. Jones, P. D. Lett, and W. D. Phillips , J. Research of the National Institute of Standards and Technology, 101, 505(1996)

[10] M.J. Holland, D. S. Jin, M. L. Chiofalo, and J. Cooper, Phys. Rev. Lett. 78, 3801(1997)

[11] Solomon, A. I. (1983). A Lie algebraic approach to order parameters. Proceedings of the Jerusalem Conference on Differential Geometric methods in Theoretical Physics, Jerusalem, D.Reidel; A. I. Solomon, "Order parameters for broken Abelian symmetries.," Annals of the New York Academy of Sciences, vol. 410, pp. 63,, 1983.

[12] M.D. Girardeau, Phys. Rev. A, 58, 775(1997)

[13] Patrick Navez, cond-mat/9804232 


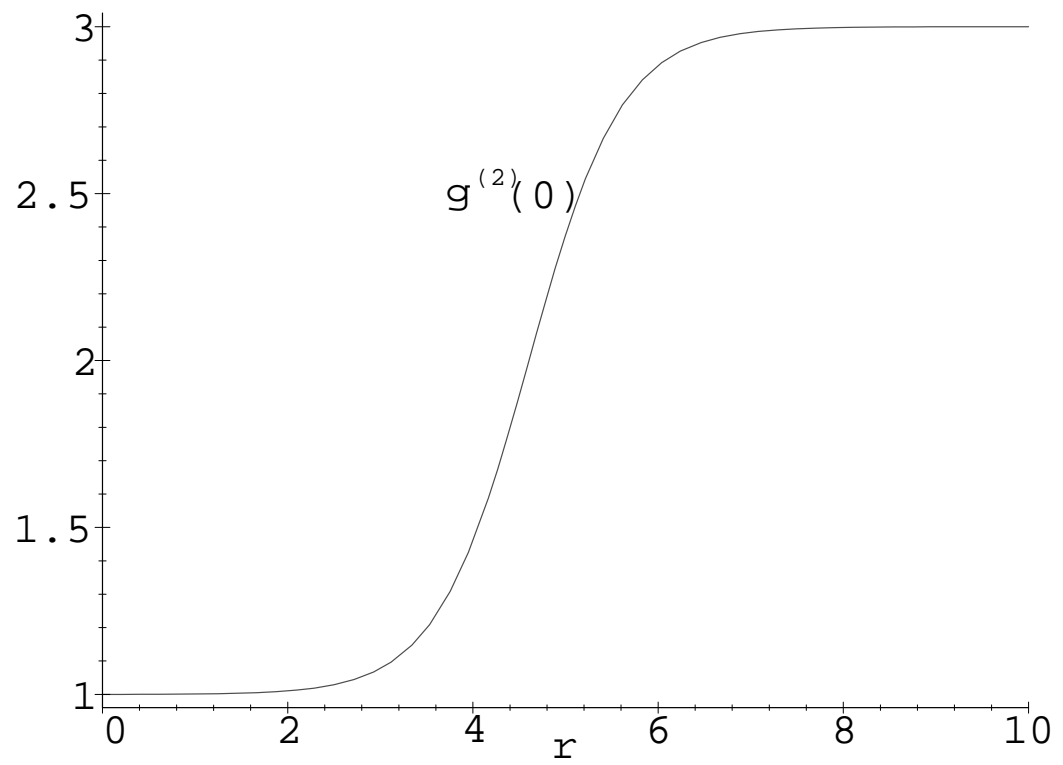

FIG. 1. The Second-order Correlation Function $g^{(2)}(0)$ for the $D S$ state $\left(\left|z_{0}\right|=50\right)$ 


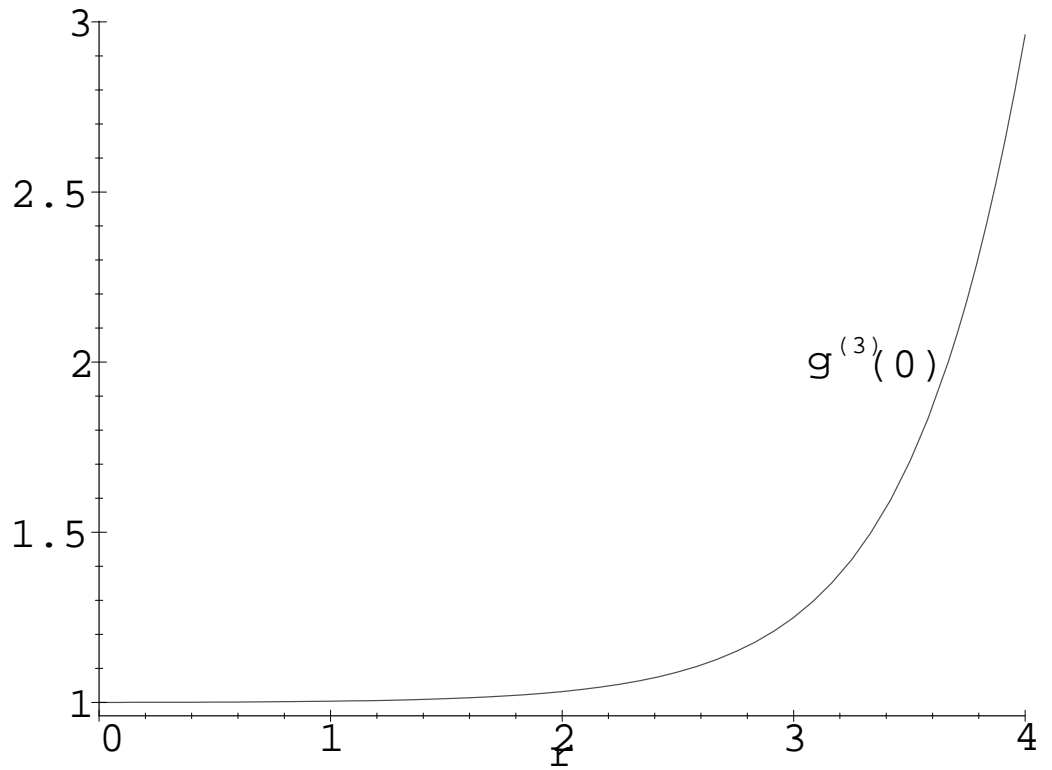

FIG. 2. The Third-order Correlation Function $g^{(3)}(0)$ for the $D S$ state $\left(\left|z_{0}\right|=50\right)$ 